\title{
Effet de l'addition de fibres de coco traitées à la potasse sur les propriétés mécaniques des matériaux de construction à base d'argile - ciment
}

\author{
Djohore Ange Christine \\ Djomo Agré Séraphin \\ Boffoue Moro Olivier \\ Emeruwa Edjikémé
}

Laboratoire de Géomatériaux et technologie du bâtiment,

UFR des sciences de la terre et des ressources minières, UFHB de Cocody

Doi: 10.19044/esj.2018.v14n36p104 URL:http://dx.doi.org/10.19044/esj.2018.v14n36p104

\begin{abstract}
Clay is a raw material commonly used for the construction of building materials, thanks to the different stabilization techniques used. These claybased materials have very improved properties when they contain potashtreated vegetable fillers. Thus, coconut fibers, agricultural waste from wad treatment, coconut palm fruit wrap (Cocos nucifra) and treated with a potassium hydroxide solution were used for making clay materials and stabilized with cement. Its objective is to study the influence of these fibers on the properties of the material. To achieve this objective, mortars composed of clays stabilized with $8 \%$ cement and fibers at different levels $(0.2,0.4,0.6$ and $0.8 \%$ ) for a constant length of $30 \mathrm{~mm}$, been developed. These different mortars with those containing no fibers were subjected to flexural and compression tests. The results obtained show that the addition of fibers improves the mechanical performance of clay mortars stabilized with $8 \%$ cement. Especially when these fibers are treated with solution of potash. The treatment of coconut fibers with the traditional potash solution made it possible to increase the maximum stress of the fiber mortar by $13 \%$ and by $5 \%$ their stiffness compared with untreated fibers. The fiber-matrix adhesion becomes stronger with fiber treatment by $8 \%$ by weight of potash.
\end{abstract}

Keywords: Mortar, Coconut fibers, Mechanical resistance, Treatment

\section{Résumé}

L'argile est une matière première couramment utilisée pour la confection de matériaux de construction, grâce aux différentes techniques de 
stabilisation employées. Ces matériaux à base d'argiles ont des propriétés très améliorées lorsqu'il contient des charges de nature végétale traitées à la potasse. C'est ainsi que des fibres de coco, déchets agricoles issues de traitement de bourres, enveloppe de fruit du cocotier (Cocos nucifra) et traitées à une solution de potasse ont été utilisées pour la confection des matériaux d'argile et stabilisée au ciment. L'objectif est d'étudier l'influence de ces fibres sur les propriétés du matériau. Pour atteindre cet objectif, des mortiers composés d'argiles stabilisées à $8 \%$ de ciment et de fibres à différentes teneurs $(0,2 ; 0,4 ; 0,6$ et $0,8 \%)$ pour une longueur constante de $30 \mathrm{~mm}$, ont été élaborés. Ces différents mortiers avec ceux ne contenant pas de fibres ont été soumis à des essais de flexion et de compression. Les résultats obtenus montrent que l'ajout de fibres améliore les performances mécaniques des mortiers d'argile stabilisée à $8 \%$ de ciment. surtout, lorsque ces fibres sont traitées avec une solution de potasse. Le traitement des fibres de coco par la solution de potasse traditionnelle a permis d'augmenter la contrainte maximale du mortier de fibres de $13 \%$ et de $5 \%$ leur rigidité par rapport à des fibres non traitées. L'adhérence fibre- matrice devient plus forte avec le traitement des fibres par $8 \%$ en masse de potasse.

Mots clés: Mortier, Fibres de coco, Résistance mécanique, Traitement

\section{Introduction}

L'Argile a démontré largement son apport bénéfique au sein d'une habitation tant ses vertus sont nombreuses. Cependant, son utilisation comme matière première dans la construction entraine un certain nombre de défauts qui fait qu'elle est délaissée au profit d'autres matières première (sable, latérite). En effet, un certain nombre de difficulté lié à l'emploi de l'argile dans la construction se pose. Il s'agit entre autre de la variation de volume (retrait et gonflement) au cours du séchage du matériau provoquant de nombreuses fissurations atténuant donc les performances mécaniques de ce dernier. $\mathrm{Ce}$ problème est généralement traité en ajoutant des stabilisants à la terre.

Les stabilisants les plus couramment utilisés sont le ciment, la chaux et le sable (Azeredo, 2005; Kouakou, 2005; Kouadio, 2010). Malgré l'apport de ces stabilisants, le problème continu d'existé d'où l'ajout d'autres additifs tels que les fibres végétales. La présence de ces fibres vont s'opposer aux retraits et aux gonflements responsable de fissures améliorant ainsi les résistances mécaniques (Ouattara, 2013 ; Tallaah, 2014 ; Millogo, 2014).

Plusieurs travaux ont montré l'efficacité des fibres végétales dans la réalisation de matériaux composites. L'incorporation de fibres végétales, matières premières abondantes et bon marché, dans une matrice à base d'argile peut être une voie intéressante pour améliorer les propriétés des mortiers de terre. Swamy et al, (1984), Ghavami et al. (1999) se sont intéressés à l'étude 
de l'incorporation de fibres de sisal et de noix de coco dans un sol fin. Ils ont constaté que les performances mécaniques sont modérées avec une amélioration de la ductilité du sol fibré par rapport au sol non fibré.

Liang (2012) a étudié l'effet de l'ajout de différentes teneurs de fibres de lin sur la résistance à la compression d'un sédiment fin stabilisé par un liant hydraulique (chaux ou ciment). Ses résultats indiquent que le sédiment mélangé avec $7 \%$ de ciment, $0,4 \%$ de lin de $17 \mathrm{~mm}$ donne une meilleure résistance à la compression. Ainsi donc, l'objectif de ce travail est de montrer que les fibres de coco peuvent être utilisées pour améliorer les performances du mortier d'argile+ ciment en vue de son utilisation comme matériaux de construction.

\section{Matériel et méthodes}

\section{Présentation de la matière première}

Sol

Le sol utilisé a été prélevé sur un site à proximité de la localité de Dabou (SW d'Abidjan) dont les coordonnées géographiques sont $5^{\circ} 19^{\prime} 70^{\prime}$ ' $\mathrm{N}$ et $4^{\circ} 22^{\prime} 80^{\prime}$ ' W. Après prélèvement et séchage, les échantillons sont réduits en poudre grâce à un broyeur à billes pendant deux heures. La poudre obtenue a servi de matière première de base dans cette étude.

\section{Ciment}

Le ciment utilisé pour cette étude est un ciment Portland composé (CPJ) sous la marque «BELIER», distribué par LAFARGEHOLCIM COTE D'IVOIRE, Société ivoirienne de ciment et de matériaux.

\section{Fibres végétales}

Les fibres végétales utilisées sont des fibres de coco extraites de la bourre de coco (enveloppe qui entoure la noix de coco). Elles sont prédécoupées en une longueur de $30 \mathrm{~mm}$ de long. Le diamètre des fibres varie de 0,1 à $1 \mathrm{~mm}$. Les fibres obtenues sont présentées sur la figure 1 .

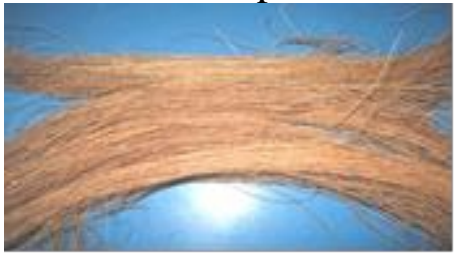

Figure 1: Fibres de coco 


\section{Caractérisation de la matière première} Analyse granulométrique

La répartition granulométrique de la poudre de terre obtenue après broyage est déterminée grâce à un granulomètre laser LS 230 de type "COULTER.

\section{Analyse par diffraction de rayon $X$}

L'analyse minéralogie du sol a été réalisée par diffraction aux rayons $\mathrm{X}$ sur la fraction fine (fraction $<2 \mu \mathrm{m}$ ). Le diffractomètre utilisé est de marque Siemens et les rayons $\mathrm{X}$ sont produits par une anticathode en cobalt de longueur d'onde 1,789 Å. Les pics ont été identifiés à l'aide du logiciel EVA.

\section{Analyse chimique}

Elle a été réalisée grâce à un spectromètre de fluorescence $\mathrm{X}$ Explorer S4 de type «Bruker», pour déterminer la composition chimique de ce sol.

\section{Traitement des fibres de coco}

Les fibres de coco sont traitées avec une solution de potasse à une concentration de $8 \%$ en masse de pastille de potasse. Le traitement a consisté à immerger les fibres dans la solution de potasse pendant $24 \mathrm{~h}$. Les fibres sont ensuite rincées à l'eau distillée afin d'éliminer l'excès de potasse. Par la suite, les fibres sont séchées à l'air ambiant du laboratoire pendant 24 heures et prêt pour l'utilisation. Les fibres brutes et traitées ont été soumises à des tests de caractérisation à savoir détermination de la masse volumique, mesure de l'absorption d'eau et résistance à la traction. Tous ces tests ont été réalisés selon les méthodes utilisées par Merzoud (2007).

\section{Procédure d'élaboration des mortiers à base de fibres et d'argile}

Les fibres de coco de $30 \mathrm{~mm}$ de longueur avec différentes teneur $(0$; $0,2 ; 0,4 ; 0,6 ; 1 \%)$ ont été mélangées à une quantité d'argile. $8 \%$ de ciment est ajouté au mélange argile plus fibres. L'ensemble est mélangé d'abord à sec. A ce mélange, une quantité d'eau a été ajoutée de telle sorte qu'elle conduise à une consistance normale dont l'affaissement au cône d'Abrams correspond à $32 \mathrm{~mm}$. La pâte obtenue est coulée dans des moules prismatiques de dimensions 4 x4 x $16 \mathrm{~cm}^{3} .72$ heures après le coulage, les échantillons sont démoulés puis conservées dans une salle à $24^{\circ} \mathrm{C}$ pendant 28 jours de séchage Après un cure de 28 jours que ces échantillons ont été soumis à un certains nombres d'essais. 


\section{Caractérisation mécanique des mortiers}

\section{Résistance à la flexion trois points et à la compression}

La résistance mécanique en flexion 3 points a été mesurée sur les éprouvettes $4 \times 4 \times 16 \mathrm{~cm}^{3}$. La résistance à la compression quant à elle, est obtenue sur les deux demi- morceaux issus de la rupture en flexion. Ces deux essais sont faits grâce à une presse hydraulique de $500 \mathrm{KN}$ de marque Control. Au cours des essais, des capteurs de forces et de déplacement sont posés sur l'échantillon. La lecture des données est faite grâce à un ordinateur. Les contraintes en flexion et en compression sont respectivement déterminées par les relations 1 et 2 :

$$
\begin{aligned}
& \sigma \mathrm{f}=(3 \mathrm{FL}) /\left(2 \mathrm{bd}^{2}\right) \\
& \sigma \mathrm{c}=\mathrm{F} /\left(\mathrm{b}^{2}\right)
\end{aligned}
$$

Où L est la distance entre les deux appuis; b est la largeur de l'échantillon; d est l'épaisseur; F, la charge maximale.

\section{Résultats et Discussion}

\section{Caractérisation de la matière première}

\section{Granulométrie de sol}

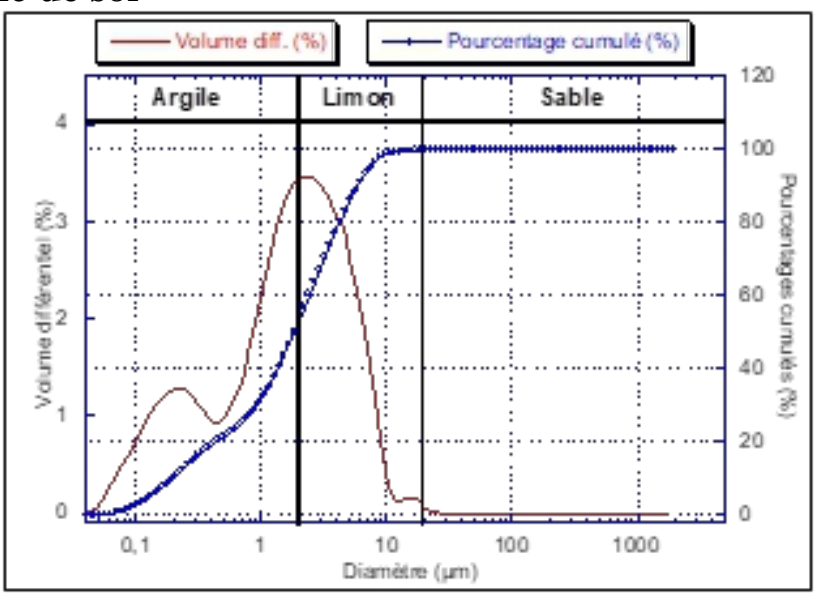

Figure 2 : Courbe d'analyse granulométrique de la terre broyée

Les résultats obtenus sont présentés sur la figure 2. La courbe granulométrique de cette poudre de terre indique qu'elle est composée de 52 $\%$ d'argile, $47 \%$ de limon et $1 \%$ de sable fin. Cette terre contenant $52 \%$ d'argile est soumise à une analyse minéralogique.

\section{Composition minéralogique}

L'analyse par diffraction de rayons $\mathrm{X}$ sur la fraction argileuse a permis de déterminer la composition minéralogique présentée sur la figure 3. 


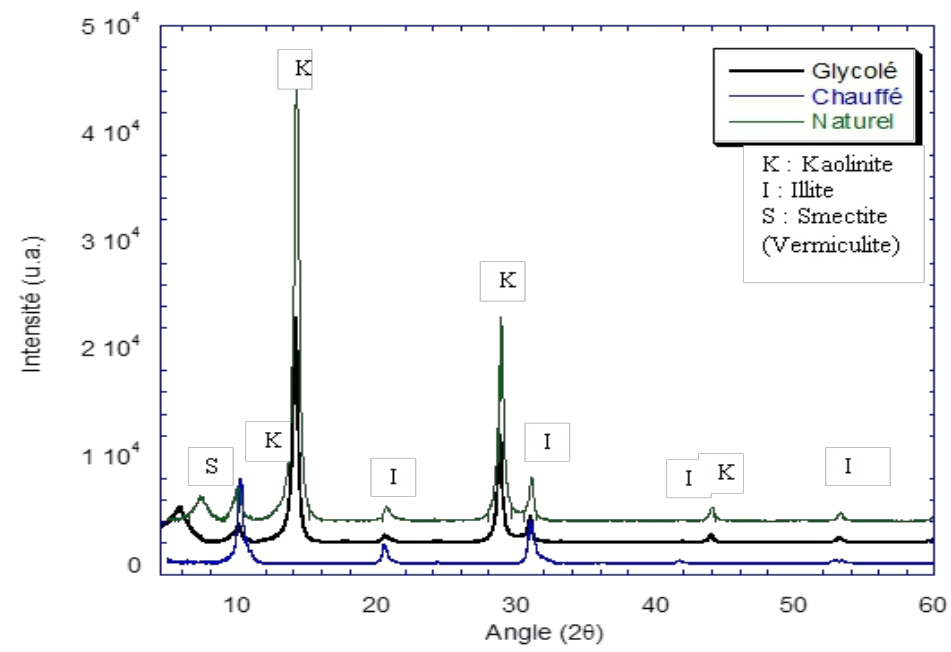

Figure 3: Diffractogramme des rayons $\mathrm{X}$ de la fraction argileuse de la terre

Elle montre que la terre contient de l'illite, de la smectite (Vermiculite) et majoritairement de la Kaolinite. Pour confirme que cette terre utilisée est une argile l'analyse chimique a été effectuée.

\section{Composition chimique}

Les résultats de l'analyse chimique sont présentés dans le tableau I.

Cette analyse montre que la terre broyée est constituée majoritairement de trois oxydes qui sont: $\mathrm{SiO} 2$ (61,9 \%); $\mathrm{Al} 2 \mathrm{O} 3$ (21,22 \%) et $\mathrm{Fe} 2 \mathrm{O} 3(4,36 \%$.)

Tableau I: Composition chimique de l'Argile

\begin{tabular}{llllllllllll}
\hline Oxydes & $\mathrm{SiO}_{2}$ & $\mathrm{Al}_{2} \mathrm{O}_{3}$ & $\mathrm{Fe}_{2} \mathrm{O}_{3}$ & $\mathrm{TiO}_{2}$ & $\mathrm{~K}_{2} \mathrm{O}$ & $\mathrm{Na}_{2} \mathrm{O}$ & $\mathrm{CaO}$ & $\mathrm{SO}_{3}$ & $\mathrm{Cr}_{2} \mathrm{O}_{3}$ & $\mathrm{MgO}$ & $\mathrm{P}_{2} \mathrm{O}_{5}$ \\
\hline Teneur $(\%)$ & 61,9 & 21,22 & 4,36 & 1,42 & 1,53 & 0,75 & 0,23 & 0,24 & 0,04 & 0,71 & 0,14 \\
\hline
\end{tabular}

Pour compléter cette analyse chimique, des caractéristiques physiques de cette terre a été également déterminées. Les différents résultats obtenus sont présentés dans le tableau II.

Ainsi la teneur élevée en silico-alumineux et la répartition granulométrique des particules de terre broyée permettent de la classer parmi les Argiles (Mekki et al., 2003).

Tableau II: Les caractéristiques physiques du sol (Kouadio, 2010)

\begin{tabular}{|c|c|c|c|}
\hline \multicolumn{2}{|c|}{$\begin{array}{r}\text { Limites d'Atterberg } \\
\end{array}$} & \multirow{2}{*}{$\begin{array}{l}\text { Surface spécifique } \\
\left(\mathrm{cm}^{2} / \mathrm{g}\right)\end{array}$} & \multirow{2}{*}{$\begin{array}{l}\text { Masse volumique } \\
\qquad\left(\mathrm{g} / \mathrm{cm}^{3}\right)\end{array}$} \\
\hline $\begin{array}{c}\text { Limite de liquidité } \mathrm{W}_{\mathrm{L}} \\
(\%)\end{array}$ & Indice de plasticité IP (\%) & & \\
\hline 50 & 27 & 3286 & 2,61 \\
\hline
\end{tabular}




\section{Caractéristiques physiques des fibres de coco}

Les résultats de la caractérisation physique et mécanique des fibres de coco non traitées et traitées sont résumées dans le tableau III.

Tableau III : Caractéristiques physiques des fibres végétales

Paramètres

Fibres de coco non traitées

Fibres de coco traitées

\begin{tabular}{lcc}
\hline Masse volumique $\left(\mathbf{g} / \mathbf{c m}^{\mathbf{3}}\right)$ & 1,25 & 1,17 \\
Absorption d'eau (\%) & 163 & 140 \\
Résistance à la traction $(\mathbf{M P a})$ & 127,2 & 142 \\
\hline
\end{tabular}

Le tableau indique une baisse de la masse volumique des fibres de coco traitées par rapport aux fibres non traitées. De même, l'absorption d'eau par les fibres traitées est moins importante que les fibres non traitées. Le tableau montré également que la résistance à la traction est améliorée lorsque les fibres sont traitées.

\section{Effet du traitement chimique sur la morphologie de la fibre}

Après immersion des fibres de coco dans une solution de $8 \%$ en masse de potasse, les surfaces des fibres ont été observées au MEB et les images obtenues sont présentées à la figure 3 .
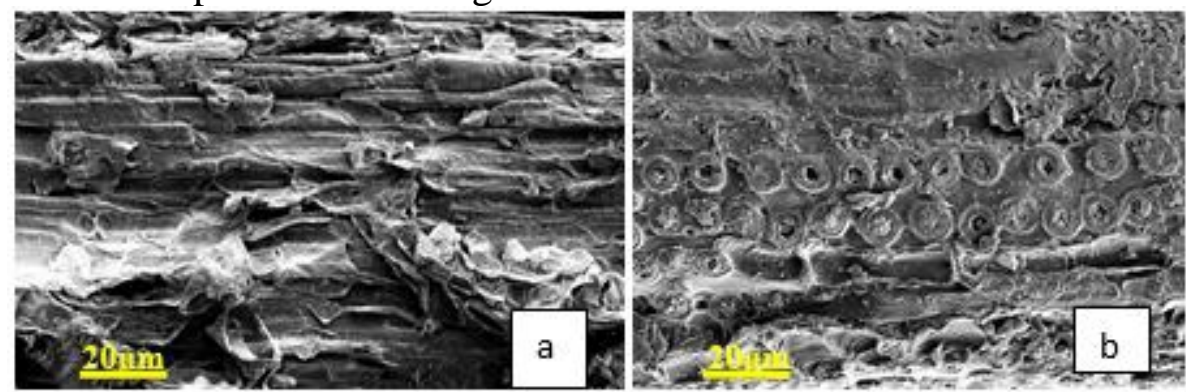

Figure 4 : Surface de la fibre de coco ; a) avant traitement, b) après traitement avec $8 \%$ en masse de potasse

La figure 4a montre que la fibre brute a en surface des impuretés, provenant probablement de cires et de graisses naturelles. Par contre, les fibres traitées à la potasse (Figure $4 \mathrm{~b}$ ) montrent une surface dépourvue d'impuretés et augmente la rugosité de la surface permettant d'améliorer l'adhérence matrice-fibre. Plusieurs auteurs notamment Sedan (2007), Le Trocdec, (2009), et autres ont en effet montrés que le traitement des fibres par des solutions basique nettoie la surface de celles-ci en dégradant les constituants amorphes, tels que les lignines, les hémicelluloses, les cires et graisses. 


\section{Comportement mécanique des mortiers à base de fibre de coco et d'argile- ciment}

\section{Effet de la teneur des fibres non traitées sur la résistance des mortiers}

Les résistances mécaniques des mortiers sans fibres et avec fibres en fonction de leur teneur ont été déterminées. Les résultats obtenus sont présentés sous forme d'histogrammes sur la figure 5 .

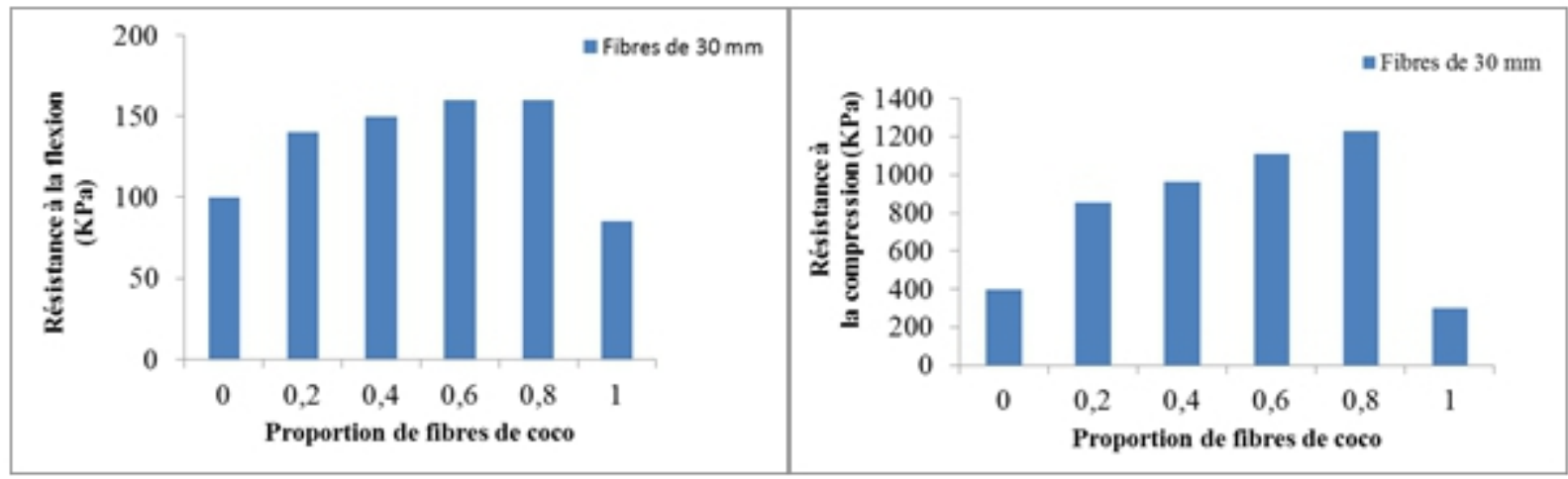

Figure 5 : Résistances mécaniques en fonction de la proportion de fibres

A $0 \%$ de fibre, les résistances à la flexion et à la compression sont respectivement de $100 \mathrm{KPa}$ et $400 \mathrm{KPa}$.

De 0,2 à $0,8 \%$ de fibres, la résistance mécanique du matériau augmente. Cette augmentation des résistances mécaniques est due au rôle joué par les fibres comme une armature reprenant les efforts transmises à la matrice (argile - ciment).

Jusqu'à $0,8 \%$ de fibre, les résistances augmentent. Cette augmentation serait due à une quantité importante de liaison fibre-matrice. Ainsi, plus le contact fibres-matrice sera nombreux, plus le transfert de charge sera de bonne qualité et plus la résistance du composite sera élevé.

Au-delà de $0,8 \%$ de fibres, les propriétés mécaniques du matériau chutent. Cette baisse est due certainement au fait que les fibres ne sont plus toutes liées les unes aux autres par la matrice. Il y'a plus des contacts fibrefibre puisque la mouillabilité des fibres par la matrice est devenue faible. Ces résultats confirment ceux de Sedan (2007) dans lesquels l'utilisation de chanvres dans un mortier à matrice cimentaire fait baisser les résistances mécaniques lorsque le taux de chanvres utilisés est élevé. Kriker (2005) aborde dans le même sens. En effet, ces études ont montrées que l'augmentation du dosage en fibres de palmier dattier fait chuter la résistance en compression.

Les résistances mécaniques maximales sont obtenues pour une proportion optimale de $0,8 \%$ de fibres en masse. A ce taux, la résistance en compression est de $1230 \mathrm{KPa}$ par rapport au mortier sans fibres qui est de 487 $\mathrm{KPa}$. 
Afin de comprendre l'influence de la proportion des fibres $(0,2,0,4$, $0,6,0,8 \%$ ) sur le comportement mécanique en compression des mortiers, les courbes contrainte déformation ont été réalisées. Elles sont présentées sur la figure 6 .

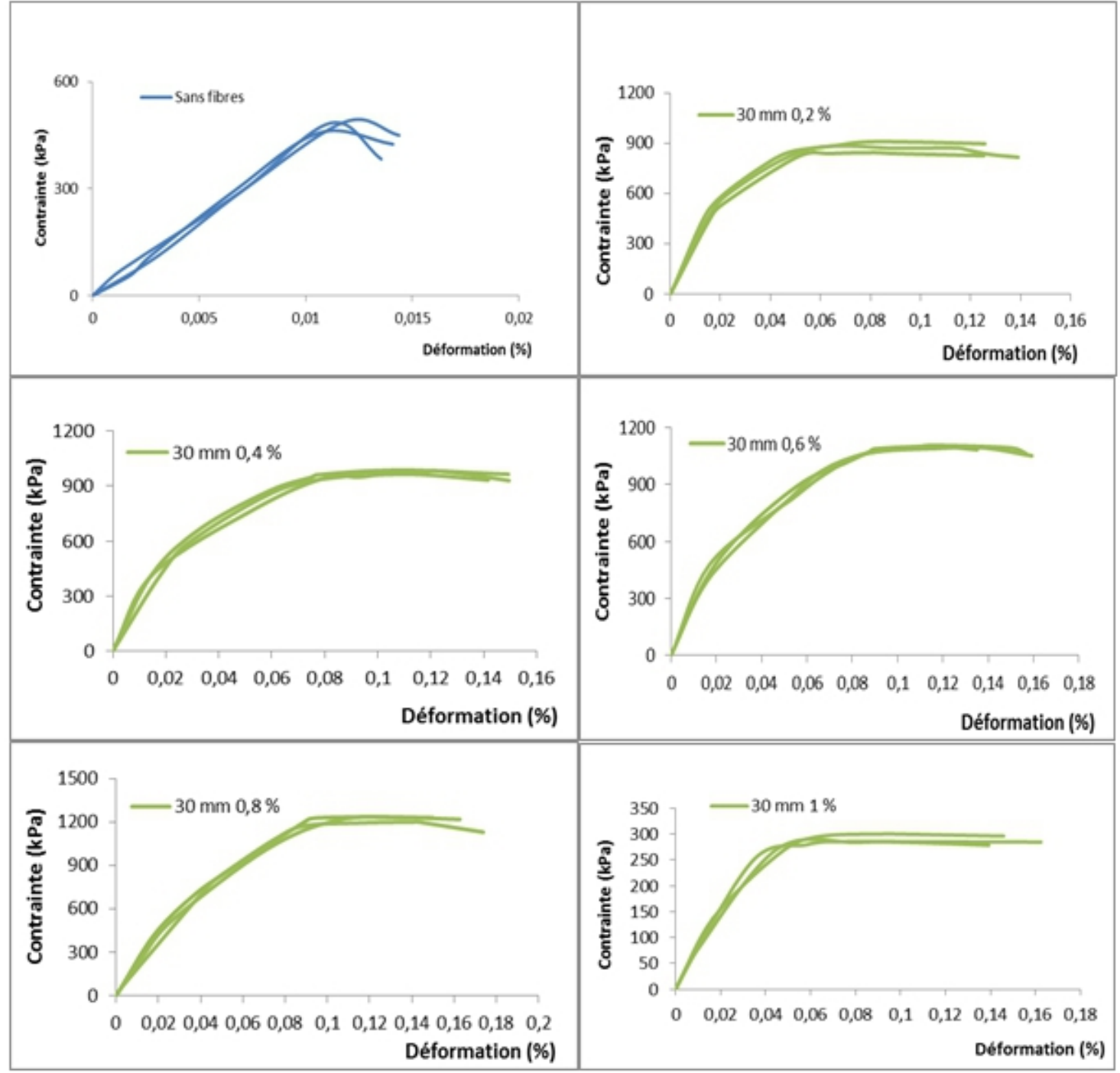

Figure 6 : Evolution des courbes contrainte-déformation des mortiers en fonction du taux de fibres de $30 \mathrm{~mm}$

La figure 6 montre que :

- la matrice minérale argile + ciment sans fibres présente un comportement élastique linéaire. Après avoir atteint sa contrainte maximale, le matériau se brise brusquement à la première fissuration (figure 7a). Sa déformation à la rupture est faible (moins de 0,2\%) et la contrainte maximale reste inférieure à $600 \mathrm{KPa}$. 
- le matériau fibré a un comportement qui présente deux phases quelques soient la teneur en fibres:

$\checkmark$ une première phase linéaire, caractérisée par un comportement élastique. Dans cette phase, le comportement du composite est principalement gouverné par celui de la matrice minérale, qui supporte la majorité des efforts. , la rupture intervient immédiatement lorsque la contrainte maximale de la matrice (argile ciment) est atteinte (figure7b) Cette phase est caractérisée par le module d'élasticité équivalent au coefficient directeur de la partie élastique de la courbe contrainte-déformation selon Mesta et al, 2002.

$\checkmark$ une deuxième phase non linéaire, caractérisée par un comportement plastique correspondant à l'effort porté par les fibres.

Puis il se produit une rupture progressive de l'interface fibres/matrice suivie d'un décollement des fibres de la matrice (figure7c).

La déformation à la rupture est plus importante avec l'augmentation de la teneur en fibres de $30 \mathrm{~mm}$ de longueur.

Plus les fibres de coco sont importantes dans la matrice, plus elles s'ancrent dans le mortier et plus grande sera leur efficacité. Cependant au-delà de $0,8 \%$, la contrainte est faible et varie peu alors que la déformation à la rupture est importante.
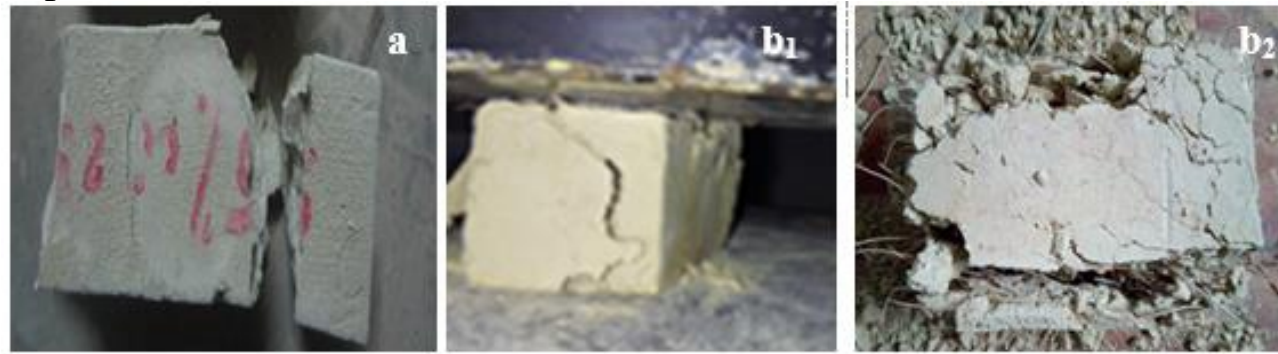

Figure 7 : Mode de rupture des éprouvettes en compression ; a) mortier sans fibres ; b) mortier contenant des fibres de coco, b1 - fissuration de la matrice argile-ciment, b2décollement des fibres de la matrice

\section{Influence du traitement des fibres sur le comportement en compression}

Les résistances mécaniques et les modules d'élasticité des éprouvettes de mortiers sans fibres et avec fibres brutes ou traitées ont été déterminés. Le tableau IV rassemble les différents résultats obtenus.

Tableau IV : Caractéristiques mécaniques en compression des différents mortiers

\begin{tabular}{|c|c|c|c|}
\hline fibres & Mortiers sans & Mortiers de fibres & $\begin{array}{c}\text { Mortier de fibres } \\
\text { traitées à } \mathrm{KOH}\end{array}$ \\
\hline $\begin{array}{c}\text { Résistance à la } \\
\text { compression }(\mathrm{KPa})\end{array}$ & 487 & 1230 & 1390 \\
\hline $\begin{array}{l}\text { Module d'élasticité } \\
\text { (MPa) }\end{array}$ & 42002 & 19414 & 30373 \\
\hline
\end{tabular}


Les résultats montrent une nette amélioration des caractéristiques mécaniques à la suite du traitement par la potasse. Le mortier de fibres traitées présente la résistance mécanique la plus élevée (1390KPa), que celle du mortier renforcé par les fibres non traités $(1230 \mathrm{KPa})$. Ce dernier a une résistance supérieure à celle du mortier sans fibres $(487 \mathrm{KPa})$.

Le traitement des fibres par $8 \%$ en masse de potasse améliore la résistance mécanique du mortier de fibres de $13 \%$ comparée à celle du mortier de fibres non traitées. Cela peut être due à l'amélioration de l'adhérence fibre-matrice minérale (Taallah, 2014 ; Fertikh, 2011). De plus, la résistance mécanique du mortier de fibres traitées est nettement plus améliorée de $186 \%$ par rapport à celle du mortier sans fibres.

Par ailleurs le module d'élasticité est plus élevé lorsque les fibres sont traitées alors qu'il est faible par rapport à celui du mortier sans fibres. Les mortiers contenant les fibres de coco traités sont plus rigide que les mortiers contenant les fibres non traitées d'où l'augmentation du module d'élasticité.

\section{Effet du traitement des fibres sur la déformation à la rupture}

La figure 8 illustre l'évolution des contrainte-déformation en compression pour $0,8 \%$ de fibres brute et traitées de $30 \mathrm{~mm}$ de long.

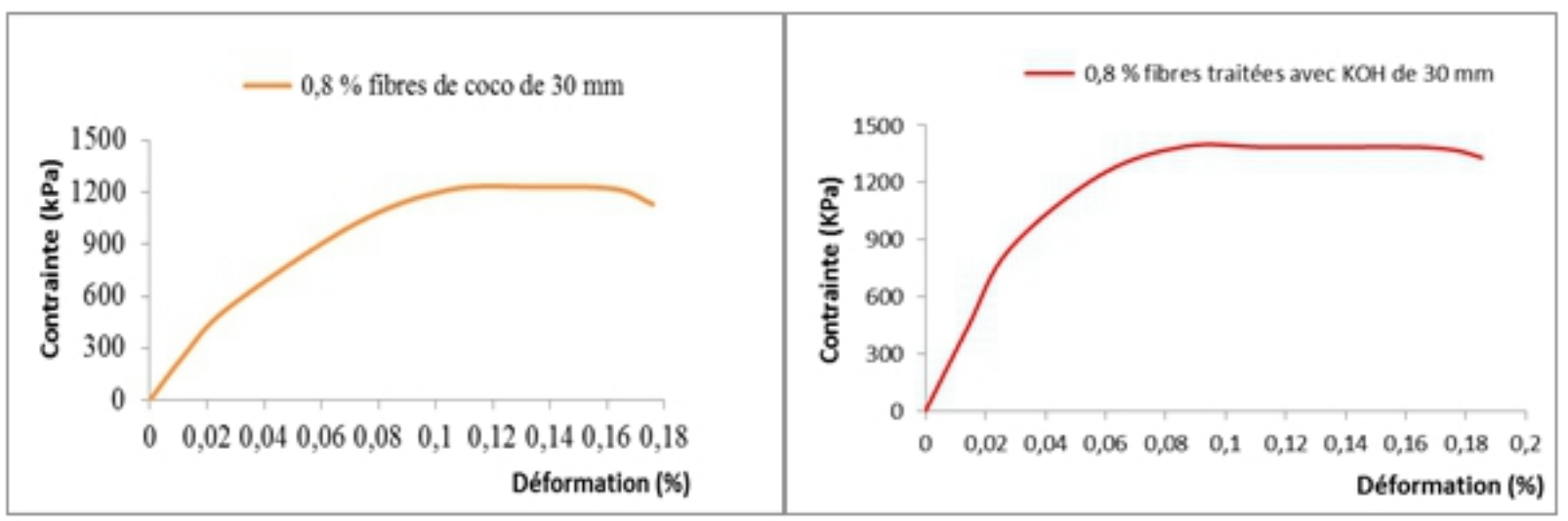

Figure 8 : Courbes contrainte-déformation des mortiers contenant les fibres de coco

A l'observation de la figure 7, les courbes contrainte - déformation peuvent être scindées en deux phases : une première phase où le comportement est élastique et la deuxième phase où le comportement est plastique. L'ampleur de plasticité des mortiers de fibres traitées est plus importante que celle du mortier de fibres non traitées.

Les fibres traitées confèrent au matériau un comportement plus ductile que les fibres non traitées.

Le traitement à la potasse des fibres de coco a permis d'augmenter la contrainte maximale du mortier de fibres de $13 \%$, comme il a permis également d'améliorer de $56 \%$ la rigidité du mortier d'argile - ciment par 
rapport à des fibres non traitées. Ces résultats sont en accord avec ceux obtenus par Le Troedec (2009). Elle a constaté que le traitement à la soude des fibres de chanvre a permis d'accroître les contraintes et les modules d'élasticités des bétons de chanvre.

\section{Conclusion}

Cette étude a permis d'étudier l'addition de fibres de coco sur le comportement mécanique des mortiers d'argile stabilisé à $8 \%$ de ciment. Les résultats obtenus montrent que l'incorporation de fibres de coco améliore les résistances mécaniques et la déformabilité des mortiers argile + ciment. Ces derniers deviennent de plus en plus ductiles avec l'augmentation de la proportion de fibres de coco. En outre la proportion de $0,8 \%$ de fibres de 30 mm permet d'avoir une résistance optimale de $1230 \mathrm{KPa}$ comparé au mortier sans fibre $(486 \mathrm{KPa})$. De plus le traitement des fibres de coco par $8 \%$ en masse de potasse a permis d'augmenter la contrainte maximale et la rigidité du matériau(d'argile $+8 \%$ ciment + fibres) par rapport à des fibres non traitées. L'adhérence entre la matrice argile+ciment et fibres de coco devient alors forte avec le traitement des fibres de coco.

\section{References:}

1. Alves De Azeredo, G., (2005). Mise au point de procedures d'essais mécaniques sur mortiers de terre: application à l'étude de leur rhéologie. Ph.D. thesis, Institut National des Sciences Appliquées, Lyon, France, $356 \mathrm{pp}$

2. Fertikh S, Merzoud M, Benazzouk A, Douzane O, Langlet T., (2011). Elaboration et caractérisation de composites à base de fibres naturelles de diss à matrice argile/chaux » Récents Progrès en Génie des Procédés, Numéro 101 - 2011ISSN 1775-335X - ISBN 2910239-75-6, Ed. SFGP, Paris, Franc

3. Ghavami K, Filho RDT, Barbosa NP (1999). Behaviour of composite soil reinforced with naturalfibres. Cem Concr Compos; 21(1):39-48, 1999.

4. Kouadio K. C. (2010). Élaboration et caractérisation de blocs d'argile stabilisée au ciment (Cimarg) : influence de l'apport de dégraissant sur les caractéristiques physiques et mécaniques des blocs. Thèse de Doctorat des Sciences de la Terre option Géomatériaux, Université de Cocody, Abidjan, 151p

5. Kouakou C. H. (2005). Valorisation des argiles de Côte d'Ivoire : étude de la stabilisation à froid de l'argile de Dabou avec un liant hydraulique (le ciment Portland). Thèse de Doctorat des Sciences de la Terre, option Géomatériaux, Université de Cocody, Abidjan 196 p. 
6. Le Trodec M. (2009). Caractérisation des interactions physicochimiques dans un matériau composite à base de phyllosilicates, de chaux et de fibres cellulosiques, Thèse de doctorat, Université de Limoge, France, $156 \mathrm{p}$

7. Liang Y. (2012). Co-valorisation de sédiments et de sols fins par apport de liants et de fibres. Université de Caen, France. Thèse de doctorat, Université de Caen, 194 p.

8. Maria Catalina N.V., Vanessa Spinosi A., Carlos A .R., Reinaldo S.(2012). Effect of the addition of coal-ash and cassava peels on the engineering properties of compressed earth blocks, Construction and building Materials 36, 276-286

9. Merzoud M (2007)., "Elaboration et caractérisation d'un matériau composite à base de fibres de diss dans la fabrication de la maçonnerie". Thèse de doctorat d'Etat, Université Badji Mokhtar, pp 123

10. Mesta Ph., Reiffsteck Ph; (2002) Modules de deformation en mécanique des sols: Définition, determination à partir des essais triaxiaux et incertitude. Paramètres de calculs geotechnique. Magan (ed), presses de l'ENPC/ LCPC, Paris,p 8

11. Ouattara S. (2013). Recherche de briques légères : conception et caractérisation de briques crues à base d'argile et sciure de bois, stabilisées au ciment portland. Thèse de Doctorat Science de la terre option Géomatériaux, Université F.H.B. d'Abidjan, 175 p.

12. Sédan D. (2007). Étude des interactions physico-chimiques aux interfaces fibres de chanvre/ciment. Influence sur les propriétés mécaniques du composite. Thèse de Doctorat, Université de Limoges, France, 129 p.

13. Swamy R. H. S, Ahuja B. M, Krishamoorthy Y. S., (1984). Behavior of concrete reinforced with jute, coir, bamboo fibers? The international journal of cement composite and light weight concrete, volume 5, p 13

14. Taallah, B. (2014). Etude du comportement physico-mécanique du bloc de terre comprimée avec fibres, Thèse de doctorat, Université Mohamed Khider-Biskra, 202 p.

15. Van Damme, H. (2013). "La terre, un béton d'argile". Pour la Science, 423, pp. 51-57.

16. Yahiaoui Lamia (2011). Caractérisation d'un composite à la rupture à base des fibres végétales (Diss) ; mémoire de MAGISTER, Universite Ferhat Abbas-Setifufas (Algerie) ; 118p. 